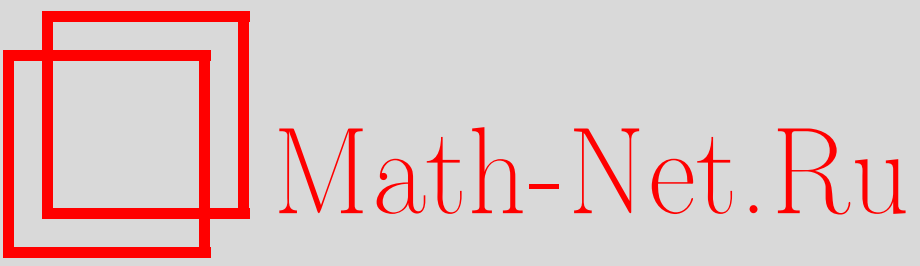

А. Б. Пичкур, Описание класса подстановок, представимых в виде произведения двух подстановок с фиксированным числом мобильных точек. II, Mатем. вопр. криптогр., 2013, том 4, выпуск 1, 87-109

DOI: https://doi.org/10.4213/mvk75

Использование Общероссийского математического портала Math-Net.Ru подразумевает, что вы прочитали и согласны с пользовательским соглашением

http: //www . mathnet.ru/rus/agreement

Параметры загрузки:

IP : 35.173 .219 .149

26 апреля 2023 г., 13:52:45 


\title{
Описание класса подстановок, представимых в виде произведения двух подстановок с фиксированным числом мобильных точек. II
}

\author{
А. Б. Пичкур \\ ООО «Центр сертификационных исследований», Москва
}

Получено 19.VII.2012

В работе полностью описано строение класса подстановок степени $N$, представимых в виде произведения двух подстановок с $q$ и $q+t$ мобильными точками, $2 \leq t<N-2,2 \leq q<\frac{N-t}{2}+1$.

Ключевые слова: симметрическая группа, мультипликативные представления, неподвижные точки

Description of the set of permutations representable as a product of two permutations with fixed number of mobile points. II

\section{A. B. Pichkur}

LLC "Certification Research Center", Moscow

Abstract. We describe completely the structure of the set of $N$-element permutations representable as the product of two permutations with $q$ and $q+t$ mobile points correspondingly, $1 \leq t<N-2,2 \leq q<\frac{N-t}{2}+1$.

Key words: symmetric group, multiplicative representations, fixed points

Citation: Mathematical Aspects of Cryptography, 2013, vol. 4, no. 1, pp. 87-109 (Russian). 
Пусть подстановка $G \in S_{N}$ - множество мобильных точек подстановки $G, 2 \leq q \leq N-$ натуральное число. Обозначим через

$$
\Gamma_{N}(q)=\left\{G \in S_{N}|| \Gamma(G) \mid=q\right\}
$$

множество всех подстановок степени $N$, имеющих ровно $q$ мобильных точек. $\Gamma(G) \subseteq \Omega=\{1, \ldots, N\}$.

В предшествующей работе [1] было полностью описано строение множества $\Gamma_{N}(q) \cdot \Gamma_{N}(q)$ при $4 \leq q \leq \frac{N}{2}$ и $N \geq 8$.

$\S 1$

Сначала опишем множество всех подстановок из $\Gamma_{N}(q) \cdot \Gamma_{N}(q+1)$, то есть всех подстановок, представимых в виде произведения двух подстановок с $q$ и $q+1$ мобильными точками.

Утверждение 1. Если $q_{1} \neq q_{2}$, то $\Gamma_{N}\left(q_{1}\right) \cdot \Gamma_{N}\left(q_{2}\right)=\Gamma_{N}\left(q_{2}\right) \cdot \Gamma_{N}\left(q_{1}\right)$.

Доказательство утверждения сразу вытекает из критерия сопряженности подстановок в $S_{N}$.

Утверждение 2. Если $N \geq 6,2 \leq q_{1}<q_{2}<N$, то имеет место включение

$$
\Gamma_{N}\left(q_{1}\right) \cdot \Gamma_{N}\left(q_{2}\right) \subseteq \Gamma_{N}\left(q_{1}+1\right) \cdot \Gamma_{N}\left(q_{2}+1\right) .
$$

Доказательство проводится по аналогии с доказательством утверждения 3 работы [1].

Пусть $H \in \Gamma_{N}\left(q_{1}\right) \cdot \Gamma_{N}\left(q_{2}\right)$, то есть имеет место равенство $H=H_{1} \cdot H_{2}$ для некоторых $H_{1} \in \Gamma_{N}\left(q_{1}\right), H_{2} \in \Gamma_{N}\left(q_{2}\right)$.

1. Если $\quad \Gamma\left(H_{1}\right) \not \subset \Gamma\left(H_{2}\right), \quad$ то $\quad$ существуют $\quad \alpha_{1} \in \Gamma\left(H_{1}\right) \backslash \Gamma\left(H_{2}\right)$ и $\beta_{1} \in \Gamma\left(H_{2}\right) \backslash \Gamma\left(H_{1}\right)$. Рассмотрим циклы из цикловой записи подстановок $H_{1}, H_{2}$, содержащие $\alpha_{1}$ и $\beta_{1}$ соответственно:

$$
\begin{aligned}
& H_{1}=\left(\alpha_{1}, \ldots, \alpha_{r}\right) \cdot H_{1}^{\prime}, \\
& H_{2}=\left(\beta_{1}, \ldots, \beta_{s}\right) \cdot H_{2}^{\prime} .
\end{aligned}
$$

Нетрудно убедиться в выполнении равенства

$$
\begin{aligned}
& \left(\left(\alpha_{1}, \ldots, \alpha_{r}, \beta_{1}\right) \cdot H_{1}{ }^{\prime}\right)\left(\left(\beta_{1}, \alpha_{1}, \beta_{2}, \ldots, \beta_{s}\right) \cdot H_{2}{ }^{\prime}\right)= \\
& =\left(\left(\alpha_{1}, \ldots, \alpha_{r}\right) \cdot H_{1}{ }^{\prime}\right)\left(\left(\beta_{1}, \ldots, \beta_{s}\right) \cdot H_{2}{ }^{\prime}\right)=H_{1} \cdot H_{2}=H .
\end{aligned}
$$


Так как

$$
\begin{aligned}
& \Gamma\left(\left(\alpha_{1}, \ldots, \alpha_{r}, \beta_{1}\right) \cdot H_{1}{ }^{\prime}\right)=q_{1}+1, \\
& \Gamma\left(\left(\beta_{1}, \alpha_{1}, \beta_{2}, \ldots, \beta_{s}\right) \cdot H_{2}{ }^{\prime}\right)=q_{2}+1,
\end{aligned}
$$

то полученное равенство означает, что $H \in \Gamma_{N}\left(q_{1}+1\right) \cdot \Gamma_{N}\left(q_{2}+1\right)$.

2. Пусть теперь $\Gamma\left(H_{1}\right) \subseteq \Gamma\left(H_{2}\right)$. В этом случае $\Gamma(H) \subseteq \Gamma\left(H_{2}\right)$ и, значит, $|\Gamma(H)| \leq q_{2}$. Из соотношения

$$
\left|\Omega \backslash\left(\Gamma\left(H_{1}\right) \cup \Gamma\left(H_{2}\right)\right)\right|=N-\left|\Gamma\left(H_{2}\right)\right|=N-q_{2} \geq 1
$$

следует, что можно выбрать точку $\delta \in \Omega \backslash \Gamma\left(H_{2}\right)$.

Рассмотрим два возможных случая.

Сначала предположим, что $\Gamma(H) \neq \Gamma\left(H_{2}\right)$. Это означает, что существует $\alpha \in \Gamma\left(H_{1}\right)$ со свойством $H(\alpha)=\alpha$. Отсюда следует, что для некоторого $\beta \neq \alpha, \beta \in \Gamma\left(H_{1}\right)$ выполняются равенства $H_{1}(\alpha)=\beta, H_{2}(\beta)=\alpha$. Тогда выберем $\delta \in \Omega \backslash \Gamma\left(H_{2}\right)$ и положим

$$
H_{1}^{\prime}(x)=\left\{\begin{array}{lrr}
\delta, & x=\alpha, \\
\beta, & x=\delta, \\
H_{1}(x), & x \in \Gamma\left(H_{1}\right) \backslash\{\alpha\},
\end{array} H_{2}^{\prime}(x)=\left\{\begin{array}{lr}
\delta, & x=\beta, \\
\alpha, & x=\delta, \\
H_{2}(x), & x \in \Gamma\left(H_{2}\right) \backslash\{\beta\} .
\end{array}\right.\right.
$$

Непосредственно проверяется, что $H=H_{1}{ }^{\prime} \cdot H_{2}{ }^{\prime}$. Так как при этом

$$
\left|\Gamma\left(H_{1}^{\prime}\right)\right|=q_{1}+1, \quad\left|\Gamma\left(H_{2}^{\prime}\right)\right|=q_{2}+1,
$$

то полученное равенство означает, что $H \in \Gamma_{N}\left(q_{1}+1\right) \cdot \Gamma_{N}\left(q_{2}+1\right)$.

Наконец, пусть $\Gamma(H)=\Gamma\left(H_{2}\right)$. Это означает, что $H_{1}(\alpha)=\beta, \beta \neq \alpha$ и $H_{2}(\beta)=\gamma, \gamma \neq \alpha, \gamma \neq \beta$ для любого $\alpha \in \Gamma\left(H_{1}\right)$. Зафиксируем некоторую точку $\alpha \in \Gamma\left(H_{1}\right)$, выберем $\delta \in \Omega \backslash \Gamma\left(H_{2}\right)$ и положим

$$
H_{1}^{\prime}(x)=\left\{\begin{array}{lrl}
\delta, & x & =\alpha, \\
\beta, & x & =\delta, \\
H_{1}(x), & x \in \Gamma\left(H_{1}\right) \backslash\{\alpha\},
\end{array} H_{2}^{\prime}(x)=\left\{\begin{array}{lr}
\delta, & x=\beta, \\
\gamma, & x=\delta, \\
H_{2}(x), & x \in \Gamma\left(H_{2}\right) \backslash\{\beta\} .
\end{array}\right.\right.
$$

Непосредственно проверяется, что $H=H_{1}^{\prime} \cdot H_{2}^{\prime}$. Так как при этом

$$
\left|\Gamma\left(H_{1}^{\prime}\right)\right|=q_{1}+1, \quad\left|\Gamma\left(H_{2}^{\prime}\right)\right|=q_{2}+1,
$$

то полученное равенство означает, что $H \in \Gamma_{N}\left(q_{1}+1\right) \cdot \Gamma_{N}\left(q_{2}+1\right)$.

Утверждение доказано полностью. 
Теорема 1. Пусть $N \geq 8,3 \leq q \leq \frac{N}{2}, G \in S_{N}$. Если $1<|\Gamma(G)| \leq 2 q-1$, то существуют подстановки $H_{1} \in \Gamma_{N}(q), H_{2} \in \Gamma_{N}(q+1)$, для которых выполняется равенство $G=H_{1} \cdot H_{2}$.

Прежде чем приступать к доказательству этой теоремы, проверим ее выполнение для небольших значений $q \in\{3,4\}$.

Сначала отметим один очевидный факт: если $H \in \Gamma_{N}(q) \cdot \Gamma_{N}(q+1)$, то любая подстановка, сопряженная с $H$, также лежит в $\Gamma_{N}(q) \cdot \Gamma_{N}(q+1)$. Значит, множество $\Gamma_{N}(q) \cdot \Gamma_{N}(q+1)$ является объединением некоторых классов сопряженных в группе $S_{N}$ подстановок.

Пусть $q=3$. Согласно теореме 1 должно выполняться включение

$$
\Gamma_{N}(2) \cup \Gamma_{N}(3) \cup \Gamma_{N}(4) \cup \Gamma_{N}(5) \subseteq \Gamma_{N}(3) \cdot \Gamma_{N}(4) .
$$

С учетом сделанного выше замечания для проверки этого свойства необходимо выписать все возможные цикловые структуры подстановок из $\Gamma_{N}(2) \cup \Gamma_{N}(3) \cup \Gamma_{N}(4) \cup \Gamma_{N}(5)$ и в каждом случае указать подстановки из $\Gamma_{N}(3), \Gamma_{N}(4)$, произведение которых дает подстановку с нужной цикловой структурой. Выпишем результаты расчетов в виде таблицы.

\begin{tabular}{|l|l|l|}
\hline & $\begin{array}{c}\text { Цикловые } \\
\text { структуры }\end{array}$ & \multicolumn{1}{|c|}{$\begin{array}{c}\text { Представление в виде произведения } \\
\text { двух подстановок из } \Gamma_{N}(3), \Gamma_{N}(4)\end{array}$} \\
\hline$\Gamma_{N}(2)$ & {$\left[2^{1}\right]$} & $(1,2,3) \cdot(1,3,4,2)=(2,4)$ \\
\hline$\Gamma_{N}(3)$ & {$\left[3^{1}\right]$} & $(1,2,3) \cdot(1,3)(2,4)=(1,4,2)$ \\
\hline \multirow{2}{*}{$\Gamma_{N}(4)$} & {$\left[2^{2}\right]$} & $(1,2,3) \cdot(1,2)(4,5)=(2,3)(4,5)$ \\
\cline { 2 - 3 } & {$\left[4^{1}\right]$} & $(1,2,3) \cdot(1,2,3,4)=(1,3,2,4)$ \\
\hline \multirow{2}{*}{$\Gamma_{N}(5)$} & {$\left[2^{1}, 3^{1}\right]$} & $(1,2,3) \cdot(1,2,4,5)=(1,4,5)(2,3)$ \\
\cline { 2 - 3 } & {$\left[5^{1}\right]$} & $(1,2,3) \cdot(1,4)(3,5)=(1,2,5,3,4)$ \\
\hline
\end{tabular}

Пусть теперь $q=4$. В силу утверждения 2

$$
\Gamma_{N}(2) \cup \Gamma_{N}(3) \cup \Gamma_{N}(4) \cup \Gamma_{N}(5) \subseteq \Gamma_{N}(3) \cdot \Gamma_{N}(4) \subseteq \Gamma_{N}(4) \cdot \Gamma_{N}(5) .
$$


Поэтому осталось проверить включение $\Gamma_{N}(6) \cup \Gamma_{N}(7) \subseteq$ $\subseteq \Gamma_{N}(4) \cdot \Gamma_{N}(5)$. Снова выпишем результаты расчетов в виде таблицы.

\begin{tabular}{|c|l|l|}
\hline & $\begin{array}{c}\text { Цикловые } \\
\text { структуры }\end{array}$ & \multicolumn{1}{|c|}{$\begin{array}{c}\text { Представление в виде произведения } \\
\text { двух подстановок из } \Gamma_{N}(5)\end{array}$} \\
\hline \multirow{5}{*}{$\Gamma_{N}(6)$} & {$\left[6^{1}\right]$} & $(1,2,3,4) \cdot(2,3,4,5,6)=(1,3,5,6,2,4)$ \\
\cline { 2 - 3 } & {$\left[2^{1}, 4^{1}\right]$} & $(1,2,3,4) \cdot(2,3,4)(5,6)=(1,3,2,4)(5,6)$ \\
\cline { 2 - 3 } & {$\left[2^{3}\right]$} & $(1,2)(4,5) \cdot(1,2,3)(6,7)=(1,3)(4,5)(6,7)$ \\
\cline { 2 - 3 } & {$\left[3^{2}\right]$} & $(1,2,3,4) \cdot(1,2)(5,6,7)=(2,3,4)(5,6,7)$ \\
\hline \multirow{5}{*}{$\Gamma_{N}(7)$} & {$\left[7^{1}\right]$} & $(1,2,3,4) \cdot(1,5,6)(2,7)=(1,7,2,3,4,5,6)$ \\
\cline { 2 - 3 } & {$\left[2^{1}, 5^{1}\right]$} & $(1,2,3,4) \cdot(1,5,6,2,7)=(2,3,4,5,6)(1,7)$ \\
\cline { 2 - 3 } & {$\left[2^{2}, 3^{1}\right]$} & $(1,2,3,4) \cdot(1,2,5)(6,7)=(1,5)(2,3,4)(6,7)$ \\
\cline { 2 - 3 } & {$\left[3^{1}, 4^{1}\right]$} & $(1,2)(3,4) \cdot(2,3)(5,6,7)=(1,3,4,2)(5,6,7)$ \\
\hline
\end{tabular}

\section{Доказательство теоремы 1}

1. Выше было показано, что теорема 1 верна при $q \in\{3,4\}$. Далее доказательство теоремы проводится индукцией по $q$ по аналогии с доказательством теоремы 4 из [1].

Пусть $q>4$. Если $G \in S_{N}$ и $|\Gamma(G)| \leq 2 q-3$, то по предположению индукции $G \in \Gamma_{N}(q-1) \cdot \Gamma_{N}(q)$. Тогда $G \in \Gamma_{N}(q) \cdot \Gamma_{N}(q+1)$ по утверждению 2.

2. Рассмотрим случай $G \in S_{N}$ и $|\Gamma(G)|=2 q-2$.

2а. Пусть в цикловой записи $G$ имеются циклы одинаковой длины

$$
G=\left(\alpha_{1}, \ldots, \alpha_{r}\right)\left(\beta_{1}, \ldots, \beta_{r}\right) G_{1}
$$

$\left|\Gamma\left(G_{1}\right)\right|=2(q-r)-2, \quad 2 \leq r \leq q-1 . \quad$ Обозначим $\quad \Omega^{\prime}=\{1, \ldots, N\} \backslash$ $\backslash\left\{\alpha_{1}, \ldots, \alpha_{r}, \beta_{1}, \ldots, \beta_{r}\right\},\left|\Omega^{\prime}\right|=N^{\prime}=N-2 r, q^{\prime}=q-r<q, G_{1} \in S\left(\Omega^{\prime}\right)$. При этом выполнено неравенство $q^{\prime} \leq \frac{N^{\prime}}{2}$. 
Если при этом $q^{\prime} \geq 3$, то по предположению индукции существуют две подстановки $H_{1}, H_{2} \in S\left(\Omega^{\prime}\right), \quad H_{1} \in \Gamma_{N^{\prime}}\left(q^{\prime}\right), \quad H_{2} \in \Gamma_{N^{\prime}}\left(q^{\prime}+1\right)$, для которых имеет место равенство $G_{1}=H_{1} \cdot H_{2}$. Тогда

$$
G=\left[\left(\alpha_{1}, \ldots, \alpha_{r}\right) \cdot H_{1}\right] \cdot\left[\left(\beta_{1}, \ldots, \beta_{r}\right) \cdot H_{2}\right],
$$

причем $\left(\alpha_{1}, \ldots, \alpha_{r}\right) \cdot H_{1} \in \Gamma_{N}(q), \quad\left(\beta_{1}, \ldots, \beta_{r}\right) \cdot H_{2} \in \Gamma_{N}(q+1) . \quad$ Значит, $G \in \Gamma_{N}(q) \cdot \Gamma_{N}(q+1)$.

Если $q^{\prime}=2$, то $\left|\Gamma\left(G_{1}\right)\right|=2$, и подстановка $G_{1}$ является транспозицией: $G_{1}=\left(\gamma_{1}, \gamma_{2}\right)$. Тогда $r=q-2$ и имеет место равенство

$$
G=\left(\alpha_{1}, \ldots, \alpha_{q-2}\right)\left(\gamma_{1}, \delta\right) \cdot\left(\beta_{1}, \ldots, \beta_{q-2}\right)\left(\gamma_{1}, \delta, \gamma_{2}\right),
$$

где $\delta \in \Omega \backslash \Gamma(G)$. Из данного равенства следует, что $G \in \Gamma_{N}(q) \cdot \Gamma_{N}(q+1)$.

Если $q^{\prime}=1$, то $G_{1}$ — тождественная подстановка, и

$$
G=\left(\alpha_{1}, \ldots, \alpha_{q-1}\right)\left(\beta_{1}, \ldots, \beta_{q-1}\right) .
$$

Тогда для любого $\delta \in \Omega \backslash \Gamma(G)$ имеем равенство

$$
G=\left(\delta, \alpha_{1}, \ldots, \alpha_{q-1}\right) \cdot\left(\delta, \alpha_{1}\right)\left(\beta_{1}, \ldots, \beta_{q-1}\right),
$$

из которого следует включение $G \in \Gamma_{N}(q) \cdot \Gamma_{N}(q+1)$.

2б. Пусть теперь в цикловой записи $G$ длины всех неединичных циклов различны. Пусть один из этих циклов имеет нечетную длину $r=2 t+1 \geq 3$ :

$$
G=\left(\alpha_{1}, \ldots, \alpha_{2 t+1}\right) G_{1},
$$

$1 \leq t<q-1, \quad\left|\Gamma\left(G_{1}\right)\right|=2(q-t-1)-1$. Обозначим $\Omega^{\prime}=\{1, \ldots, N\} \backslash\left\{\alpha_{1}, \ldots, \alpha_{2 t+1}\right\}$, $\left|\Omega^{\prime}\right|=N^{\prime}=N-2 t-1, q^{\prime}=q-t-1<q, G_{1} \in S\left(\Omega^{\prime}\right)$. При этом выполнено неравенство $q^{\prime} \leq \frac{N}{2}-t-1=\frac{N-2 t-2}{2}=\frac{N^{\prime}-1}{2}<\frac{N^{\prime}}{2}$.

Непосредственно проверяется, что

$$
\left(\alpha_{1}, \ldots, \alpha_{2 t+1}\right)=\left(\alpha_{1}, \ldots, \alpha_{t+1}\right) \cdot\left(\alpha_{1}, \alpha_{t+2}, \ldots, \alpha_{2 t+1}\right) \in \Gamma_{N}(t+1) \cdot \Gamma_{N}(t+1) .
$$

Если при этом $q^{\prime} \geq 3$, то по предположению индукции существуют подстановки $H_{1}, H_{2} \in S\left(\Omega^{\prime}\right), H_{1} \in \Gamma_{N^{\prime}}\left(q^{\prime}\right), H_{2} \in \Gamma_{N^{\prime}}\left(q^{\prime}+1\right)$, для которых выполняется равенство $G_{1}=H_{1} \cdot H_{2}$. Тогда

$$
G=\left[\left(\alpha_{1}, \ldots, \alpha_{t+1}\right) \cdot H_{1}\right] \cdot\left[\left(\alpha_{1}, \alpha_{t+2}, \ldots, \alpha_{2 t+1}\right) \cdot H_{2}\right],
$$


причем

$$
\begin{gathered}
\left(\alpha_{1}, \ldots, \alpha_{t+1}\right) \cdot H_{1} \in \Gamma_{N}(q-t-1+t+1)=\Gamma_{N}(q), \\
\left(\alpha_{1}, \alpha_{t+2}, \ldots, \alpha_{2 t+1}\right) \cdot H_{2} \in \Gamma_{N}(q-t-1+t+1+1)=\Gamma_{N}(q+1) .
\end{gathered}
$$

Если $q^{\prime}=2$, то $t=q-3$. Следовательно, $\left|\Gamma\left(G_{1}\right)\right|=3$, и $G_{1}=\left(\gamma_{1}, \gamma_{2}, \gamma_{3}\right)$. Тогда для любого $\delta \in \Omega \backslash \Gamma(G)$ имеем равенство

$$
G=\left(\delta, \alpha_{1}, \ldots, \alpha_{q-1}\right) \cdot\left(\alpha_{1}, \delta, \alpha_{q}, \ldots, \alpha_{2 q-5}\right)\left(\gamma_{1}, \gamma_{2}, \gamma_{3}\right) .
$$

Осталось заметить, что

$$
\begin{gathered}
\left(\delta, \alpha_{1}, \ldots, \alpha_{q-1}\right) \in \Gamma_{N}(q) \\
\left(\alpha_{1}, \delta, \alpha_{q}, \ldots, \alpha_{2 q-5}\right)\left(\gamma_{1}, \gamma_{2}, \gamma_{3}\right) \in \Gamma_{N}(q-2+3)=\Gamma_{N}(q+1) .
\end{gathered}
$$

В случае $q^{\prime}=1$ получаем противоречие: $\left|\Gamma\left(G_{1}\right)\right|=1$.

2в. Пусть в цикловой записи $G$ длины всех неединичных циклов различны и четны. Пусть один из этих циклов имеет длину $2 t$ :

$$
G=\left(\alpha_{1}, \ldots, \alpha_{2 t}\right) G_{1},
$$

$1 \leq t \leq q-1, \quad\left|\Gamma\left(G_{1}\right)\right|=2(q-t)-2 . \quad$ Обозначим $\quad \Omega^{\prime}=\{1, \ldots, N\} \backslash\left\{\alpha_{1}, \ldots, \alpha_{2 t}\right\}$, $\left|\Omega^{\prime}\right|=N^{\prime}=N-2 t, q^{\prime}=q-t<q, G_{1} \in S\left(\Omega^{\prime}\right)$. При этом выполнено неравенство $q^{\prime} \leq \frac{N}{2}-t=\frac{N-2 t}{2}=\frac{N^{\prime}}{2}$.

Поскольку $q>4$ и длины всех неединичных циклов $G$ различны, то можно выбрать цикл $\left(\alpha_{1}, \ldots, \alpha_{2 t}\right)$ с условием $t \geq 2$. Непосредственно проверяется, что

$$
\left(\alpha_{1}, \ldots, \alpha_{2 t}\right)=\left(\alpha_{1}, \ldots, \alpha_{t}\right) \cdot\left(\alpha_{1}, \alpha_{t+1}, \ldots, \alpha_{2 t}\right) \in \Gamma_{N}(t) \cdot \Gamma_{N}(t+1) .
$$

Если при этом $q^{\prime} \geq 4$, то по теореме 4 из [1] существуют подстановки $H_{1}, H_{2} \in S\left(\Omega^{\prime}\right), \quad H_{1}, H_{2} \in \Gamma_{N^{\prime}}\left(q^{\prime}\right), \quad$ для которых выполняется равенство $G_{1}=H_{1} \cdot H_{2}$. Тогда

$$
G=\left[\left(\alpha_{1}, \ldots, \alpha_{t}\right) \cdot H_{1}\right] \cdot\left[\left(\alpha_{1}, \alpha_{t+1}, \ldots, \alpha_{2 t}\right) \cdot H_{2}\right],
$$

причем

$$
\begin{gathered}
\left(\alpha_{1}, \ldots, \alpha_{t}\right) \cdot H_{1} \in \Gamma_{N}(q-t+t)=\Gamma_{N}(q) \\
\left(\alpha_{1}, \alpha_{t+1}, \ldots, \alpha_{2 t}\right) \cdot H_{2} \in \Gamma_{N}(q-t+t+1)=\Gamma_{N}(q+1) .
\end{gathered}
$$


Если $q^{\prime}=3$, то $t=q-3$. Следовательно, $\left|\Gamma\left(G_{1}\right)\right|=4$, и возможны только два случая: $\left[G_{1}\right]=\left[2^{2}\right]$ или $\left[G_{1}\right]=\left[4^{1}\right]$. В первом случае

$$
G=\left(\beta_{1}, \beta_{2}\right)\left(\gamma_{1}, \gamma_{2}\right)\left(\alpha_{1}, \ldots, \alpha_{2 t}\right)=\left(\beta_{1}, \beta_{2}\right) \cdot G_{2},
$$

где $\left|\Gamma\left(G_{2}\right)\right|=2(q-1)-2$. Так как $q>4$, то $q-1>3$, и по предположению индукции $G_{2} \in \Gamma_{N-2}(q-1) \cdot \Gamma_{N-2}(q)$. Тогда $G=\left(\beta_{1}, \beta_{2}\right) \cdot G_{2} \in \Gamma_{N}(q+1) \cdot \Gamma_{N}(q)$. Осталось воспользоваться утверждением 1 .

Во втором случае $G=\left(\beta_{1}, \beta_{2}, \beta_{3}, \beta_{4}\right)\left(\alpha_{1}, \ldots, \alpha_{2 t}\right)$. Непосредственно проверяется равенство

$$
G=\left(\alpha_{1}, \ldots, \alpha_{q}\right) \cdot\left(\alpha_{1}, \alpha_{q+1}, \ldots, \alpha_{2 t}\right)\left(\beta_{1}, \beta_{2}, \beta_{3}, \beta_{4}\right),
$$

причем

$\left(\alpha_{1}, \ldots, \alpha_{q}\right) \in \Gamma_{N}(q),\left(\alpha_{1}, \alpha_{q+1}, \ldots, \alpha_{2 t}\right)\left(\beta_{1}, \beta_{2}, \beta_{3}, \beta_{4}\right) \in \Gamma_{N}(q-5+4)=\Gamma_{N}(q-1)$. Итак, $G \in \Gamma_{N}(q) \cdot \Gamma_{N}(q-1)$. Далее, $G \in \Gamma_{N}(q-1) \cdot \Gamma_{N}(q)$ по утверждению 1 и $G \in \Gamma_{N}(q) \cdot \Gamma_{N}(q+1)$ по утверждению 2.

Если $q^{\prime}=2$, то $t=q-2$. Следовательно, $\left|\Gamma\left(G_{1}\right)\right|=2$, и $G_{1}$ является транспозицией: $G=\left(\beta_{1}, \beta_{2}\right)\left(\alpha_{1}, \ldots, \alpha_{2 t}\right)$. Так как $\left|\Gamma\left(\left(\alpha_{1}, \ldots, \alpha_{2 t}\right)\right)\right|=2(q-2)=$ $=2(q-1)-2$ и $q-1>3$, то по предположению индукции имеем включение $\left(\alpha_{1}, \ldots, \alpha_{2 t}\right) \in \Gamma_{N}(q-1) \cdot \Gamma_{N}(q)$. Тогда $G=\left(\beta_{1}, \beta_{2}\right)\left(\alpha_{1}, \ldots, \alpha_{2 t}\right) \in \Gamma_{N}(q+1) \cdot \Gamma_{N}(q)$. Осталось воспользоваться утверждением 1 .

В случае $q^{\prime}=1$ получаем условие $\left|\Gamma\left(G_{1}\right)\right|=0$. Значит, $G=\left(\alpha_{1}, \ldots, \alpha_{2 t}\right)$ и $t=q-1>3$. Включение $\left(\alpha_{1}, \ldots, \alpha_{2 t}\right) \in \Gamma_{N}(t) \cdot \Gamma_{N}(t+1)=\Gamma_{N}(q-1) \cdot \Gamma_{N}(q)$ доказано выше. Тогда по утверждению 2 имеем включение $G \in \Gamma_{N}(q) \cdot \Gamma_{N}(q+1)$.

3. Наконец, рассмотрим случай $G \in S_{N}$ и $|\Gamma(G)|=2 q-1$.

3а. Пусть в цикловой записи $G$ имеются циклы одинаковой длины:

$$
\begin{gathered}
G=\left(\alpha_{1}, \ldots, \alpha_{r}\right)\left(\beta_{1}, \ldots, \beta_{r}\right) G_{1}, \\
\left|\Gamma\left(G_{1}\right)\right|=2(q-r)-1,2 \leq r<q-1 .
\end{gathered}
$$

Снова обозначим $\Omega^{\prime}=\{1, \ldots, N\} \backslash\left\{\alpha_{1}, \ldots, \alpha_{r}, \beta_{1}, \ldots, \beta_{r}\right\},\left|\Omega^{\prime}\right|=N^{\prime}=N-2 r$, $q^{\prime}=q-r<q, G_{1} \in S\left(\Omega^{\prime}\right)$. При этом выполнено неравенство $q^{\prime} \leq \frac{N^{\prime}}{2}$. 
Если при этом $q^{\prime} \geq 3$, то по предположению индукции существуют две подстановки $H_{1}, H_{2} \in S\left(\Omega^{\prime}\right), H_{1} \in \Gamma_{N^{\prime}}\left(q^{\prime}\right), H_{2} \in \Gamma_{N^{\prime}}\left(q^{\prime}+1\right)$, для которых имеет место равенство $G_{1}=H_{1} \cdot H_{2}$. Тогда

$$
G=\left[\left(\alpha_{1}, \ldots, \alpha_{r}\right) \cdot H_{1}\right] \cdot\left[\left(\beta_{1}, \ldots, \beta_{r}\right) \cdot H_{2}\right],
$$

причем $\left(\alpha_{1}, \ldots, \alpha_{r}\right) \cdot H_{1} \in \Gamma_{N}(q),\left(\beta_{1}, \ldots, \beta_{r}\right) \cdot H_{2} \in \Gamma_{N}(q+1)$.

Если $q^{\prime}=2$, то $q-r=2,\left|\Gamma\left(G_{1}\right)\right|=3$ и $G_{1}=\left(\gamma_{1}, \gamma_{2}, \gamma_{3}\right)$. Тогда

$$
\begin{aligned}
& G=\left(\alpha_{1}, \ldots, \alpha_{q-2}\right)\left(\beta_{1}, \ldots, \beta_{q-2}\right)\left(\gamma_{1}, \gamma_{2}, \gamma_{3}\right)= \\
& =\left(\gamma_{1}, \gamma_{2}, \alpha_{1}, \ldots, \alpha_{q-2}\right) \cdot\left(\alpha_{1}, \gamma_{3}, \gamma_{1}\right)\left(\beta_{1}, \ldots, \beta_{q-2}\right),
\end{aligned}
$$

причем $\left(\gamma_{1}, \gamma_{2}, \alpha_{1}, \ldots, \alpha_{q-2}\right) \in \Gamma_{N}(q),\left(\alpha_{1}, \gamma_{3}, \gamma_{1}\right)\left(\beta_{1}, \ldots, \beta_{q-2}\right) \in \Gamma_{N}(q+1)$.

3б. Осталось рассмотреть случай, когда $|\Gamma(G)|=2 q-1$ и все неединичные циклы подстановки $G$ имеют различную длину. Пусть $G$ является произведением $r$ циклов, длины которых равны $1<l_{1}<l_{2}<\ldots<l_{r}, \sum_{i=1}^{r} l_{i}=2 q-1$. Тогда $G$ сопряжена с подстановкой

$$
\tilde{G}=\left(1, \ldots, l_{1}\right)\left(l_{1}+1, \ldots, l_{1}+l_{2}\right) \ldots\left(l_{1}+\ldots+l_{r-1}+1, \ldots, 2 q-1\right) .
$$

Если $r=1$, то при $q \geq 5$ подстановка

$$
\begin{gathered}
(1,2, \ldots, q) \cdot[(1, q+1, \ldots, 2 q-2)(2,2 q-1)]= \\
=(1,2 q-1,2, \ldots, q, q+1, \ldots, 2 q-2),
\end{gathered}
$$

с одной стороны, лежит в $\Gamma_{N}(q) \cdot \Gamma_{N}(q+1)$, а с другой стороны, сопряжена с $\tilde{G}$. Значит, в рассматриваемом случае $G \in \Gamma_{N}(q) \cdot \Gamma_{N}(q+1)$.

Пусть теперь $r \geq 2$. Тогда существует такое $2 \leq s \leq r$, что

$$
\sum_{i=1}^{s-1} l_{i}<q, \quad \sum_{i=1}^{s} l_{i} \geq q
$$

Умножим подстановку $\tilde{G}$ слева на цикл $X=\left(l_{1}, l_{1}+l_{2}, \ldots, l_{1}+\ldots+l_{s-1}\right)$ длины $s-1$, а справа - на цикл $Y=\left(l_{1}+\ldots+l_{s-1}+1, l_{1}+\ldots+l_{s}+1, \ldots, l_{1}+\ldots+l_{r-1}+1\right)$ длины $r-s+1$. Если $s-1$ или $r-s+1$ равны единице, то соответствующие циклы равны тождественной подстановке. В результате имеем подстановку

$$
\begin{gathered}
H=X \cdot \tilde{G} \cdot Y= \\
=\left(1,2, \ldots, l_{1}, l_{1}+1, \ldots, l_{1}+l_{2}, \ldots, l_{1}+\ldots+l_{s-1}\right)\left(l_{1}+\ldots+l_{s-1}+1, \ldots ., 2 q-1\right) .
\end{gathered}
$$


Обозначим $l_{1}+\ldots+l_{s-1}+1=m \leq q$. Можно заметить, что подстановка $H$ представима в виде произведения

$$
H=(1,2, \ldots, q) \cdot(m, 1, q+1, \ldots, 2 q-1) \in \Gamma_{N}(q) \cdot \Gamma_{N}(q+1) .
$$

Тогда

$$
\begin{gathered}
\tilde{G}=X^{-1} \cdot H \cdot Y^{-1}=\left[\left(l_{1}, l_{1}+l_{2}, \ldots, l_{1}+\ldots+l_{s-1}\right)^{-1} \cdot(1,2, \ldots, q)\right] \times \\
\times\left[(m, 1, q+1, \ldots, 2 q-1) \cdot\left(l_{1}+\ldots+l_{s-1}+1, l_{1}+\ldots+l_{s}+1, \ldots, l_{1}+\ldots+l_{r-1}+1\right)^{-1}\right] .
\end{gathered}
$$

Рассмотрим подстановки

$$
\begin{gathered}
T_{1}=\left(l_{1}, l_{1}+l_{2}, \ldots, l_{1}+\ldots+l_{s-1}\right)^{-1} \cdot(1,2, \ldots, q), \\
T_{2}=(m, 1, q+1, \ldots, 2 q-1) \cdot\left(m, l_{1}+\ldots+l_{s}+1, \ldots, l_{1}+\ldots+l_{r-1}+1\right)^{-1} .
\end{gathered}
$$

Непосредственно убеждаемся, что

$$
\begin{gathered}
T_{1}=\left(l_{1}, l_{1}+l_{2}, \ldots, l_{1}+\ldots+l_{s-1}\right)^{-1} \cdot(1,2, \ldots, q)= \\
=\left(1,2, \ldots, l_{1}, l_{1}+\ldots+l_{s-1}+1, \ldots, q\right)\left(l_{1}+1, \ldots, l_{1}+l_{2}\right) \ldots\left(l_{1}+\ldots+l_{s-2}+1, \ldots, l_{1}+\ldots+l_{s-1}\right) .
\end{gathered}
$$

Значит, $T_{1} \in \Gamma_{N}(q)$. (Если $s=2$, то $T_{1}=(1,2, \ldots, q)$ также лежит в $\Gamma_{N}(q)$.)

Так как $\sum_{i=1}^{s} l_{i} \geq q$, то $\sum_{i=1}^{s} l_{i}+1 \geq q+1$.

Если $\sum_{i=1}^{s} l_{i}+1=q+1$, то

$$
\begin{gathered}
T_{2}=(m, 1, q+1, \ldots, 2 q-1) \cdot\left(m, q+1, \ldots, l_{1}+\ldots+l_{r-1}+1\right)^{-1}= \\
=(1, m)\left(q+1, \ldots, l_{1}+\ldots+l_{s+1}\right)\left(l_{1}+\ldots+l_{s+1}+1, \ldots, l_{1}+\ldots+l_{s+2}\right) \ldots \\
\ldots\left(l_{1}+\ldots+l_{r-1}+1, \ldots, 2 q-1\right) .
\end{gathered}
$$

При этом подстановка $T_{2}$ имеет

$$
2+l_{s+1}+\ldots+l_{r}=2 q-1-\left(l_{1}+\ldots+l_{s}\right)+2=2 q-1-q+2=q+1
$$

мобильных точек, то есть $T_{2} \in \Gamma_{N}(q+1)$.

$$
\begin{gathered}
\text { Пусть теперь } \sum_{i=1}^{s} l_{i}+1>q+1 . \text { Тогда } \\
T_{2}=(m, 1, q+1, \ldots, 2 q-1) \cdot\left(m, l_{1}+\ldots+l_{s}+1, \ldots, l_{1}+\ldots+l_{r-1}+1\right)^{-1}= \\
=\left(m, 1, q+1, \ldots, l_{1}+\ldots+l_{s}\right)\left(l_{1}+\ldots+l_{s}+1, \ldots, l_{1}+\ldots+l_{s+1}\right) \ldots\left(l_{1}+\ldots+l_{r-1}+1, \ldots, 2 q-1\right) .
\end{gathered}
$$


При этом подстановка $T_{2}$ имеет

$$
2+\left(l_{1}+\ldots+l_{s}-q\right)+l_{s+1}+\ldots+l_{r}=2 q-1-q+2=q+1
$$

мобильных точек, то есть $T_{2} \in \Gamma_{N}(q+1)$.

Итак, во всех возможных случаях доказано, что любая подстановка $G \in \Gamma_{N}(2 q-1)$ принадлежит множеству $\Gamma_{N}(q) \cdot \Gamma_{N}(q+1)$.

Теорема 1 доказана полностью.

Следствие. При $N \geq 8,3 \leq q \leq \frac{N}{2}$

$$
\Gamma_{N}(q) \cdot \Gamma_{N}(q+1) \supseteq \bigcup_{r=2}^{2 q-1} \Gamma_{N}(r)
$$

Замечание. Очевидно, что при $q=2$ утверждение следствия не выполняется. Действительно, множество $\Gamma_{N}(2)$ состоит из всех транспозиций, а множество $\Gamma_{N}(3)-$ из всех циклов длины 3. Поэтому множество $\Gamma_{N}(2) \cdot \Gamma_{N}(3)$ состоит только из нечетных подстановок. Следовательно, в нем не содержится множество $\Gamma_{N}(3)$.

Далее рассмотрим, какие подстановки из множеств $\Gamma_{N}(2 q), \quad \Gamma_{N}(2 q+1)$ принадлежат произведению $\Gamma_{N}(q) \cdot \Gamma_{N}(q+1)$. Пусть $G$ является произведением $r$ неединичных циклов, длины которых равны $m_{1}, m_{2}, \ldots, m_{r}$.

Утверждение 3. Пусть $N \geq 4,2 \leq q<\frac{N}{2}$, подстановка $G \in \Gamma_{N}(2 q+1)$ является произведением $r$ неединичных ијиклов, длины которых равны $m_{1}, m_{2}, \ldots, m_{r}, \quad \sum_{i=1}^{r} m_{i}=2 q+1$. Подстановка $G$ принадлежсит множеству $\Gamma_{N}(q) \cdot \Gamma_{N}(q+1)$ в том и только в том случае, когда существует такое подмножество $\left\{i_{1}, \ldots, i_{k}\right\} \subseteq\{1, \ldots, r\}$, что $m_{i_{1}}+\ldots+m_{i_{k}}=q$.

Доказательство. Если для некоторых подстановок $H_{1} \in \Gamma_{N}(q)$, $H_{2} \in \Gamma_{N}(q+1)$ выполняется равенство $G=H_{1} \cdot H_{2}$, то $\Gamma\left(H_{1}\right) \cap \Gamma\left(H_{2}\right)=\varnothing$. Отсюда следует, что подстановки $H_{1}, H_{2}$ являются произведениями некоторых циклов из цикловой записи подстановки $G$. Пусть для подстановки $H_{1}$ длины этих циклов равны $m_{i_{1}}, \ldots, m_{i_{k}}$. Поскольку $H_{1} \in \Gamma_{N}(q)$, то $m_{i_{1}}+\ldots+m_{i_{k}}=q$. 
Обратно, пусть искомое подмножество $\left\{i_{1}, \ldots, i_{k}\right\}$ существует. Обозначим $\left\{j_{1}, \ldots, i_{r-k}\right\} \subseteq\{1, \ldots, r\} \backslash\left\{i_{1}, \ldots, i_{k}\right\}$. Тогда $m_{j_{1}}+\ldots+m_{j_{r-k}}=2 q+1-q=q+1$. Построим подстановку $H_{1}$ как произведение циклов подстановки $G$, имеющих длины $m_{i_{1}}, \ldots, m_{i_{k}}$, а подстановку $H_{2}$ как произведение циклов подстановки $G$, имеющих длины $m_{j_{1}}, \ldots, m_{j_{r-k}}$. Тогда $H_{1} \in \Gamma_{N}(q), H_{2} \in \Gamma_{N}(q+1)$ и $G=H_{1} \cdot H_{2}$.

Замечание. Из доказанного утверждения вытекает, что $\Gamma_{N}(2 q+1) \not \subset$ $\not \subset \Gamma_{N}(q) \cdot \Gamma_{N}(q+1)$. Действительно, $(2 q+1)$-циклы лежат в $\Gamma_{N}(2 q+1)$, однако они не удовлетворяют условию утверждения 4.

Утверждение 4. Пусть $N \geq 4,2 \leq q \leq \frac{N}{2}$, подстановка $G \in \Gamma_{N}(2 q)$ является произведением $r$ неединичных ииклов, длины которых равны $m_{1}, m_{2}, \ldots, m_{r}, \quad \sum_{i=1}^{r} m_{i}=2 q$. Подстановка $G \quad$ принадлежит множеству $\Gamma_{N}(q) \cdot \Gamma_{N}(q+1)$ в том и только в том случае, когда выполнено условие: существуют такое $i_{0} \in\{1, \ldots, r\}$ и такое подмножество $\left\{i_{1}, \ldots, i_{k}\right\} \subseteq\{1, \ldots, r\} \backslash\left\{i_{0}\right\}$, что $m_{i_{0}}>2 u q-m_{i_{1}}+m_{i_{2}} \ldots+m_{i_{k}} \in\left\{2, \ldots, m_{i_{0}}-1\right\}$.

Доказательство. Если для некоторых подстановок $H_{1} \in \Gamma_{N}(q)$, $H_{2} \in \Gamma_{N}(q+1)$ выполняется равенство $G=H_{1} \cdot H_{2}$, то $\left|\Gamma\left(H_{1}\right) \cap \Gamma\left(H_{2}\right)\right|=1$. Пусть $\Gamma\left(H_{1}\right) \cap \Gamma\left(H_{2}\right)=\left\{\alpha_{1}\right\}$. Отсюда следует, что в подстановке $H_{1}$ существует цикл $\left(\alpha_{1}, \ldots, \alpha_{u}\right)$, в подстановке $H_{2}$ существует цикл $\left(\alpha_{1}, \beta_{2}, \ldots, \beta_{v}\right)$, а остальные циклы в $H_{1}, H_{2}$ являются циклами из цикловой записи подстановки $G$.

Пусть для подстановки $H_{1}$ длины этих циклов равны $m_{i_{1}}, \ldots, m_{i_{k}}$. Поскольку $H_{1} \in \Gamma_{N}(q)$, то $m_{i_{1}}+\ldots+m_{i_{k}}=q-u$. При вычислении произведения $H_{1} \cdot H_{2}$ получится цикл длины $u+v-1$

$$
\left(\alpha_{1}, \ldots, \alpha_{u}\right)\left(\alpha_{1}, \beta_{2, \ldots, \beta_{v}}\right)=\left(\alpha_{1}, \alpha_{2}, \ldots, \alpha_{u}, \beta_{2,}, \ldots, \beta_{v}\right),
$$

причем этот цикл есть в цикловой записи подстановки $G$. Тогда выберем такое $i_{0} \in\{1, \ldots, r\}$, что $m_{i_{0}}=u+v-1$. Поскольку $u \geq 2, v \geq 2$, то $m_{i_{0}}>2$. Кроме того, $\left\{i_{1}, \ldots, i_{k}\right\} \subseteq\{1, \ldots, r\} \backslash\left\{i_{0}\right\}$ и $q-\left(m_{i_{1}}+\ldots+m_{i_{k}}\right)=u \in\left\{2, \ldots, m_{i_{0}}-1\right\}$. 
Обратно, пусть искомое $i_{0} \in\{1, \ldots, r\}$ и подмножество $\left\{i_{1}, \ldots, i_{k}\right\}$ существует. Пусть $\left\{j_{1}, \ldots, j_{r-k-1}\right\} \subseteq\{1, \ldots, r\} \backslash\left\{i_{0}, i_{1}, \ldots, i_{k}\right\}$. Тогда

$$
\begin{gathered}
q-\left(m_{i_{1}}+\ldots+m_{i_{k}}\right)=u \in\left\{2, \ldots, m_{i_{0}}-1\right\}, \\
m_{j_{1}}+\ldots+m_{j_{r-k-1}}=2 q-m_{i_{0}}-\left(m_{i_{1}}+\ldots+m_{i_{k}}\right)=2 q-m_{i_{0}}-q+u=q-m_{i_{0}}+u .
\end{gathered}
$$

Обозначим $v=m_{i_{0}}+1-u \in\left\{2, \ldots, m_{i_{0}}-1\right\}$. Тогда цикл $\left(\alpha_{1}, \ldots, \alpha_{m_{i_{0}}}\right)$ из цикловой записи подстановки $G$ можно представить в виде произведения двух циклов

$$
\left(\alpha_{1}, \ldots, \alpha_{m_{i 0}}\right)=\left(\alpha_{1}, \ldots, \alpha_{u}\right)\left(\alpha_{1}, \alpha_{u+1} \ldots, \alpha_{m_{i 0}}\right)
$$

длин $u$ и $v$ соответственно.

Построим подстановку $H_{1}$ как произведение циклов подстановки $G$, имеющих длины $m_{i_{1}}, \ldots, m_{i_{k}}$, и цикла $\left(\alpha_{1}, \ldots, \alpha_{u}\right)$. Подстановку $H_{2}$ построим как произведение циклов подстановки $G$, имеющих длины $m_{j_{1}}, \ldots, m_{j_{r-k-1}}$, и цикла $\left(\alpha_{1}, \alpha_{u+1} \ldots, \alpha_{m_{0}}\right)$. Тогда равенство $G=H_{1} \cdot H_{2}$ выполнено, подстановка $H_{1}$ имеет $m_{i_{1}}+\ldots+m_{i_{k}}+u=q$ мобильных точек, а подстановка $H_{2}$ имеет $m_{j_{1}}+\ldots+m_{j_{r-k-1}}+v=q+1$ мобильных точек.

Замечание. Из доказанного утверждения вытекает, что $\Gamma_{N}(2 q) \not \subset$ $\not \subset \Gamma_{N}(q) \cdot \Gamma_{N}(q+1)$. Рассмотрим подстановки из $\Gamma_{N}(2 q)$, являющиеся произведением двух циклов длины $q: m_{1}=q$ и $m_{2}=q$. Покажем, что такие подстановки не могут удовлетворять условию утверждения 4.

Если $i_{0}=1$, то в случае $\left\{i_{1}, \ldots, i_{k}\right\}=\{2\}$ имеем противоречие

$$
q-m_{i_{1}}+\ldots+m_{i_{k}}=q-q=0 \notin\left\{2, \ldots, m_{i_{0}}-1\right\} .
$$

В случае $\left\{i_{1}, \ldots, i_{k}\right\}=\varnothing$ снова имеем противоречие

$$
q-m_{i_{1}}+\ldots+m_{i_{k}}=q \notin\left\{2, \ldots, m_{i_{0}}-1\right\} .
$$

Случай $i_{0}=2$ рассматривается аналогично.

Итак, в теореме 1, утверждениях 3, 4 полностью описано строение множества $\Gamma_{N}(q) \cdot \Gamma_{N}(q+1)$ при $3 \leq q \leq \frac{N}{2}$. 


\section{$\S 2$}

Теперь приступим к описанию строения множества $\Gamma_{N}(q) \cdot \Gamma_{N}(q+t)$ при $t \geq 2$. Прежде всего, заметим, что для всех $r<t$

$$
\Gamma_{N}(q) \cdot \Gamma_{N}(q+t) \cap \Gamma_{N}(r)=\varnothing .
$$

Теорема 2. Пусть $N>10,2 \leq t<N-2, \quad 2 \leq q<\frac{N-t}{2}+1, \quad G \in S_{N}$. Если $t \leq|\Gamma(G)| \leq 2 q+t-2$, то существуют подстановки $H_{1} \in \Gamma_{N}(q)$, $H_{2} \in \Gamma_{N}(q+t)$, для которых выполняется равенство $G=H_{1} \cdot H_{2}$.

Доказательство теоремы проведем индукцией по $q$ и разобьем на ряд лемм.

Лемма 1. Пусть $N \geq 4,2 \leq t \leq N-2, G \in S_{N}$. Если $t \leq|\Gamma(G)| \leq t+2$, то существуют подстановки $H_{1} \in \Gamma_{N}(2), H_{2} \in \Gamma_{N}(t+2)$, для которых выполняется равенство $\mathrm{G}=\mathrm{H}_{1} \cdot \mathrm{H}_{2}$.

Доказательство. Пусть $|\Gamma(G)|=t$. Выберем $\alpha, \beta \notin \Gamma(G)$ и положим $H_{1}=(\alpha, \beta) \in \Gamma_{N}(2), H_{2}=G \cdot(\alpha, \beta) \in \Gamma_{N}(t+2)$. Тогда равенство $G=H_{1} \cdot H_{2}$ выполнено.

Пусть $|\Gamma(G)|=t+1$ и $G=\left(\alpha_{1}, \ldots, \alpha_{r}\right) \ldots\left(\beta_{1}, \ldots, \beta_{s}\right)$. Выберем $\gamma \notin \Gamma(G)$ и положим $H_{1}=\left(\alpha_{1}, \gamma\right) \in \Gamma_{N}(2), H_{2}=\left(\alpha_{1}, \gamma, \alpha_{2}, \ldots, \alpha_{r}\right) \ldots\left(\beta_{1}, \ldots, \beta_{s}\right) \in \Gamma_{N}(t+2)$. Равенство $G=H_{1} \cdot H_{2}$ снова выполнено.

Пусть $|\Gamma(G)|=t+2$. Если при этом $G$ является циклом $G=\left(\alpha_{1}, \ldots, \alpha_{t+2}\right)$, то длина этого цикла не меньше 4 . Тогда

$$
G=\left(\alpha_{1}, \alpha_{3}\right) \cdot\left[\left(\alpha_{2}, \alpha_{3}\right)\left(\alpha_{1}, \alpha_{4}, \ldots, \alpha_{t+2}\right)\right] \in \Gamma_{N}(2) \cdot \Gamma_{N}(t+2) .
$$

Если же $G$ не является циклом, то $G=\left(\alpha_{1}, \ldots, \alpha_{r}\right) \ldots\left(\beta_{1}, \ldots, \beta_{s}\right)$, и тогда

$$
G=\left(\alpha_{1}, \beta_{1}\right) \cdot\left[\left(\alpha_{1}, \beta_{2}, \ldots, \beta_{s}, \beta_{1}, \alpha_{2}, \ldots, \alpha_{r}\right) \ldots\right] \in \Gamma_{N}(2) \cdot \Gamma_{N}(t+2) .
$$

Лемма 2. Пусть $N \geq 6,2 \leq t \leq N-4, G \in S_{N}$. Если $t \leq|\Gamma(G)| \leq t+4$, то существуют подстановки $H_{1} \in \Gamma_{N}(3), H_{2} \in \Gamma_{N}(t+3)$, для которых выполняется равенство $G=H_{1} \cdot H_{2}$.

Доказательство. Пусть $|\Gamma(G)|=t$. Выберем $\alpha, \beta, \gamma \notin \Gamma(G)$ и положим $H_{1}=(\alpha, \beta, \gamma) \in \Gamma_{N}(3), H_{2}=G \cdot(\alpha, \gamma, \beta) \in \Gamma_{N}(t+3)$. Тогда равенство $G=H_{1} \cdot H_{2}$ выполнено. 
Пусть $|\Gamma(G)|=t+1$ и $G=\left(\alpha_{1}, \ldots, \alpha_{r}\right) \ldots\left(\beta_{1}, \ldots, \beta_{s}\right)$. Выберем $\gamma, \delta \notin \Gamma(G)$ и положим $\quad H_{1}=\left(\alpha_{1}, \gamma, \delta\right) \in \Gamma_{N}(3), \quad H_{2}=\left(\alpha_{1}, \delta, \gamma, \alpha_{2}, \ldots, \alpha_{r}\right) \ldots\left(\beta_{1}, \ldots, \beta_{s}\right) \in$ $\in \Gamma_{N}(t+3)$. Равенство $G=H_{1} \cdot H_{2}$ снова выполнено.

Пусть $|\Gamma(G)|=t+2$. Выберем $\gamma \notin \Gamma(G)$. Если при этом $G$ является циклом $G=\left(\alpha_{1}, \ldots, \alpha_{t+2}\right)$, то длина этого цикла не меньше 4 , и тогда

$$
G=\left(\alpha_{1}, \gamma, \alpha_{2}\right) \cdot\left[\left(\alpha_{1}, \alpha_{3}, \ldots, \alpha_{t+2}\right)\left(\alpha_{2}, \gamma\right)\right] \in \Gamma_{N}(3) \cdot \Gamma_{N}(t+3) .
$$

Если же $G$ не является циклом, то $G=\left(\alpha_{1}, \ldots, \alpha_{r}\right) \ldots\left(\beta_{1}, \ldots, \beta_{s}\right)$, и тогда

$$
G=\left(\alpha_{1}, \gamma, \beta_{1}\right) \cdot\left[\left(\alpha_{1}, \beta_{2}, \ldots, \beta_{s}, \beta_{1}, \gamma, \alpha_{2}, \ldots, \alpha_{r}\right) \ldots\right] \in \Gamma_{N}(3) \cdot \Gamma_{N}(t+3) .
$$

Пусть $|\Gamma(G)|=t+3$. Если при этом в $G$ существует цикл $\left(\alpha_{1}, \ldots, \alpha_{r}\right)$, длина которого не меньше 3 , то

$$
G=\left(\alpha_{1}, \alpha_{3}, \alpha_{2}\right) \cdot\left[\left(\alpha_{1}, \alpha_{3}, \alpha_{2}, \ldots, \alpha_{r}\right) \ldots\right] \in \Gamma_{N}(3) \cdot \Gamma_{N}(t+3) .
$$

Если же $G$ является произведением транспозиций, то в силу условия $|\Gamma(G)|=t+3 \geq 5$ таких транспозиций должно быть не менее 3: $G=\left(\alpha_{1}, \alpha_{2}\right)\left(\beta_{1}, \beta_{2}\right)\left(\gamma_{1}, \gamma_{2}\right) \ldots$ Тогда

$$
G=\left(\alpha_{1}, \gamma_{1}, \beta_{1}\right) \cdot\left[\left(\alpha_{1}, \beta_{2}, \beta_{1}, \gamma_{2}, \gamma_{1}, \alpha_{2}\right) \ldots\right] \in \Gamma_{N}(3) \cdot \Gamma_{N}(t+3) .
$$

Пусть $|\Gamma(G)|=t+4$. Если при этом $G$ является циклом $G=\left(\alpha_{1}, \ldots, \alpha_{t+4}\right)$, то длина этого цикла не меньше 6 , и тогда

$$
G=\left(\alpha_{1}, \alpha_{3}, \alpha_{t+4}\right) \cdot\left[\left(\alpha_{4}, \ldots, \alpha_{t+4}\right)\left(\alpha_{2}, \alpha_{3}\right)\right] \in \Gamma_{N}(3) \cdot \Gamma_{N}(t+3) .
$$

Если же $G$ не является циклом, то $G=\left(\alpha_{1}, \ldots, \alpha_{r}\right) \ldots\left(\beta_{1}, \ldots, \beta_{s}\right)$, и тогда

$$
G=\left(\alpha_{1}, \alpha_{2}, \beta_{1}\right) \cdot\left[\left(\alpha_{1}, \beta_{2}, \ldots, \beta_{s}, \beta_{1}, \alpha_{3}, \ldots, \alpha_{r}\right) \ldots\right] \in \Gamma_{N}(3) \cdot \Gamma_{N}(t+3) .
$$

Лемма 2 доказана.

Лемма 3. Пусть $N \geq 8,2 \leq t \leq N-6, G \in S_{N}$. Если $t \leq|\Gamma(G)| \leq t+6$, то существуют подстановки $H_{1} \in \Gamma_{N}(4), H_{2} \in \Gamma_{N}(t+4)$, для которых выполняется равенство $G=H_{1} \cdot H_{2}$.

Доказательство. Пусть $|\Gamma(G)|=t$. Выберем $\alpha, \beta, \gamma, \delta \notin \Gamma(G)$ и положим $H_{1}=(\alpha, \beta, \gamma, \delta) \in \Gamma_{N}(4), H_{2}=G \cdot(\alpha, \delta, \gamma, \beta) \in \Gamma_{N}(t+4)$. Тогда равенство $G=H_{1} \cdot H_{2}$ выполнено. 
1. Пусть $|\Gamma(G)|=t+1$ и $G=\left(\alpha_{1}, \ldots, \alpha_{r}\right) \ldots$ Выберем $\beta, \gamma, \delta \notin \Gamma(G)$ и положим $H_{1}=\left(\alpha_{1}, \beta, \gamma, \delta\right) \in \Gamma_{N}(4), H_{2}=\left(\alpha_{1}, \delta, \gamma, \beta, \alpha_{2}, \ldots, \alpha_{r}\right) \ldots \in \Gamma_{N}(t+4)$. Равенство $G=H_{1} \cdot H_{2}$ снова выполнено.

2. Пусть $|\Gamma(G)|=t+2$. Выберем $\gamma, \delta \notin \Gamma(G)$. Если при этом $G$ является циклом $G=\left(\alpha_{1}, \ldots, \alpha_{t+2}\right)$, то длина этого цикла не меньше 4 , и тогда

$$
G=\left(\alpha_{1}, \alpha_{3}\right)(\gamma, \delta,) \cdot\left[\left(\alpha_{1}, \alpha_{4}, \ldots, \alpha_{t+2}\right)\left(\alpha_{2}, \alpha_{3}\right)(\gamma, \delta,)\right] \in \Gamma_{N}(4) \cdot \Gamma_{N}(t+4) .
$$

Если же $G$ не является циклом, то $G=\left(\alpha_{1}, \ldots, \alpha_{r}\right) \ldots\left(\beta_{1}, \ldots, \beta_{s}\right)$, и тогда

$$
G=\left(\alpha_{1}, \gamma, \delta, \beta_{1}\right) \cdot\left[\left(\alpha_{1}, \beta_{2}, \ldots, \beta_{s}, \beta_{1}, \delta, \gamma, \alpha_{2}, \ldots, \alpha_{r}\right) \ldots\right] \in \Gamma_{N}(4) \cdot \Gamma_{N}(t+4) .
$$

3. Пусть $|\Gamma(G)|=t+3$. Выберем $\delta \notin \Gamma(G)$. Если при этом в $G$ существует цикл $\left(\alpha_{1}, \ldots, \alpha_{r}\right)$, длина которого не меньше 3 , то

$$
G=\left(\alpha_{1}, \alpha_{3}, \alpha_{2}, \delta\right) \cdot\left[\left(\alpha_{1}, \delta, \alpha_{3}, \alpha_{2}, \ldots, \alpha_{r}\right) \ldots\right] \in \Gamma_{N}(4) \cdot \Gamma_{N}(t+4) .
$$

Если же $G$ является произведением транспозиций, то в силу условия $|\Gamma(G)|=t+3 \geq 5$ таких транспозиций должно быть не менее 3 : $G=\left(\alpha_{1}, \alpha_{2}\right)\left(\beta_{1}, \beta_{2}\right)\left(\gamma_{1}, \gamma_{2}\right) \ldots$ Тогда

$$
G=\left(\alpha_{1}, \gamma_{1}, \beta_{1}, \delta\right) \cdot\left[\left(\alpha_{1}, \delta, \beta_{2}, \beta_{1}, \gamma_{2}, \gamma_{1}, \alpha_{2}\right) \ldots\right] \in \Gamma_{N}(4) \cdot \Gamma_{N}(t+4) .
$$

4. Пусть $|\Gamma(G)|=t+4$. Если при этом $G$ является циклом $G=\left(\alpha_{1}, \ldots, \alpha_{t+4}\right)$, то длина этого цикла не меньше 6 , и тогда

$$
G=\left(\alpha_{1}, \alpha_{3}\right)\left(\alpha_{2}, \alpha_{4}\right) \cdot\left[\left(\alpha_{1}, \alpha_{4}, \alpha_{3}, \alpha_{2}, \alpha_{5}, \ldots, \alpha_{t+4}\right)\right] \in \Gamma_{N}(4) \cdot \Gamma_{N}(t+4) .
$$

Если же $G$ не является циклом, то $G=\left(\alpha_{1}, \ldots, \alpha_{r}\right) \ldots\left(\beta_{1}, \ldots, \beta_{s}\right)$, и тогда

$$
G=\left(\alpha_{1}, \beta_{1}\right)\left(\alpha_{2}, \beta_{2}\right) \cdot\left[\left(\alpha_{1}, \beta_{2}, \alpha_{3}, \ldots, \alpha_{r}\right) \ldots\left(\beta_{1}, \alpha_{2}, \beta_{3}, \ldots, \beta_{s}\right)\right] \in \Gamma_{N}(4) \cdot \Gamma_{N}(t+4) \text {. }
$$

5. Пусть $|\Gamma(G)|=t+5$. Если при этом $G$ является циклом $G=\left(\alpha_{1}, \ldots, \alpha_{t+5}\right)$, то длина этого цикла не меньше 7 , и тогда

$$
G=\left(\alpha_{1}, \alpha_{2}, \alpha_{5}, \alpha_{4}\right) \cdot\left(\alpha_{1}, \alpha_{5}, \alpha_{3}, \alpha_{4}, \alpha_{6}, \ldots, \alpha_{t+5}\right) \in \Gamma_{N}(4) \cdot \Gamma_{N}(t+4) .
$$

Пусть $G$ не является циклом $G=\left(\alpha_{1}, \ldots, \alpha_{r}\right) \ldots\left(\beta_{1}, \ldots, \beta_{s}\right)$ и $r \geq 3$. Тогда

$$
G=\left(\beta_{1}, \beta_{2}, \alpha_{2}, \alpha_{1}\right) \cdot\left[\left(\alpha_{1}, \alpha_{3}, \ldots, \alpha_{r}\right) \ldots\left(\beta_{1}, \alpha_{2}, \beta_{3}, \ldots, \beta_{s}\right)\right] \in \Gamma_{N}(4) \cdot \Gamma_{N}(t+4) .
$$


Наконец, пусть $G$ является произведением транспозиций. Тогда в силу условия $|\Gamma(G)|=t+5 \geq 7$ таких транспозиций должно быть не менее 4 : $G=\left(\alpha_{1}, \alpha_{2}\right)\left(\beta_{1}, \beta_{2}\right)\left(\gamma_{1}, \gamma_{2}\right)\left(\delta_{1}, \delta_{2}\right) \ldots$ Тогда

$$
G=\left(\alpha_{1}, \gamma_{1}, \beta_{2}, \beta_{1}\right) \cdot\left[\left(\alpha_{1}, \beta_{2}, \gamma_{2}, \gamma_{1}, \alpha_{2}\right)\left(\delta_{1}, \delta_{2}\right) \ldots\right] \in \Gamma_{N}(4) \cdot \Gamma_{N}(t+4) .
$$

6. Пусть $|\Gamma(G)|=t+6$. Если при этом $G$ является циклом $G=\left(\alpha_{1}, \ldots, \alpha_{t+6}\right)$, то длина этого цикла не меньше 8, и тогда

$$
G=\left(\alpha_{1}, \alpha_{2}\right)\left(\alpha_{3}, \alpha_{4}\right) \cdot\left(\alpha_{1}, \alpha_{3}, \alpha_{5}, \alpha_{6}, \ldots, \alpha_{t+6}\right) \in \Gamma_{N}(4) \cdot \Gamma_{N}(t+4) .
$$

Пусть $G$ не является циклом $G=\left(\alpha_{1}, \ldots, \alpha_{r}\right) \ldots\left(\beta_{1}, \ldots, \beta_{s}\right)$. Если при этом $r \geq 3, s \geq 3$, то

$$
G=\left(\alpha_{1}, \alpha_{2}\right)\left(\beta_{1}, \beta_{2}\right) \cdot\left[\left(\alpha_{1}, \alpha_{3}, \ldots, \alpha_{r}\right) \ldots\left(\beta_{1}, \beta_{3}, \ldots, \beta_{s}\right)\right] \in \Gamma_{N}(4) \cdot \Gamma_{N}(t+4) .
$$

Если среди неединичных циклов подстановки $G$ только один цикл не является транспозицией и при этом $r \geq 4$, то

$$
G=\left(\alpha_{1}, \alpha_{2}\right)\left(\alpha_{3}, \alpha_{4}\right) \cdot\left[\left(\alpha_{1}, \alpha_{3}, \alpha_{5}, \ldots, \alpha_{r}\right) \ldots\left(\beta_{1}, \ldots, \beta_{s}\right)\right] \in \Gamma_{N}(4) \cdot \Gamma_{N}(t+4) .
$$

Если в подстановке $G$ не является транспозицией только один цикл длины 3 , то в силу условия $|\Gamma(G)|=t+6 \geq 8$ число транспозиций в подстановке $G$ должно быть не менее 3: $G=\left(\alpha_{1}, \alpha_{2}, \alpha_{3}\right)\left(\beta_{1}, \beta_{2}\right)\left(\gamma_{1}, \gamma_{2}\right)\left(\delta_{1}, \delta_{2}\right) \ldots$ Тогда

$$
G=\left(\alpha_{1}, \gamma_{1}\right)\left(\beta_{1}, \beta_{2}\right) \cdot\left[\left(\alpha_{1}, \gamma_{2}, \gamma_{1}, \alpha_{2}, \alpha_{3}\right)\left(\delta_{1}, \delta_{2}\right) \ldots\right] \in \Gamma_{N}(4) \cdot \Gamma_{N}(t+4) .
$$

Наконец, пусть $G$ является произведением транспозиций. Тогда в силу условия $|\Gamma(G)|=t+6 \geq 8$ таких транспозиций должно быть не менее 4: $G=\left(\alpha_{1}, \alpha_{2}\right)\left(\beta_{1}, \beta_{2}\right)\left(\gamma_{1}, \gamma_{2}\right)\left(\delta_{1}, \delta_{2}\right) \ldots$ Тогда

$$
G=\left(\alpha_{1}, \alpha_{2}\right)\left(\gamma_{1}, \beta_{1}\right) \cdot\left[\left(\beta_{1}, \gamma_{2}, \gamma_{1}, \beta_{2}\right)\left(\delta_{1}, \delta_{2}\right) \ldots\right] \in \Gamma_{N}(4) \cdot \Gamma_{N}(t+4) .
$$

Лемма 3 доказана.

Из лемм 1, 2, 3 вытекает, что теорема 2 выполняется для $q \in\{2,3,4\}$.

Пусть теперь $q>4$. Если $G \in S_{N}$ и $t \leq|\Gamma(G)| \leq 2 q+t-4$, то по предположению индукции $G \in \Gamma_{N}(q-1) \cdot \Gamma_{N}(q-1+t)$. Тогда

$$
q+t-1<\frac{N-t}{2}+1+t-1=\frac{N+t}{2}<N,
$$

и $G \in \Gamma_{N}(q) \cdot \Gamma_{N}(q+t)$ по утверждению 2. 
Осталось рассмотреть случай $G \in S_{N}$ и $|\Gamma(G)| \in\{2 q+t-3,2 q+t-2\}$. Исходя из очевидных неравенств

$$
\begin{aligned}
& t+2 \leq 2 q+t-3 \leq 2(q-1)+(t+2)-2, \\
& t+2 \leq 2 q+t-2 \leq 2(q-1)+(t+2)-2,
\end{aligned}
$$

по предположению индукции получаем включение $G \in \Gamma_{N}(q-1)$. $\cdot \Gamma_{N}(q-1+t+2)$, или $G \in \Gamma_{N}(q-1) \cdot \Gamma_{N}(q+t+1)$.

Лемма 4. Пусть $N>10, \quad 2 \leq t<N-2, \quad 4<q<\frac{N-t}{2}+1, \quad G \in S_{N}$ $u|\Gamma(G)| \in\{2 q+t-3,2 q+t-2\}$. Тогда существуют подстановки $H_{1} \in \Gamma_{N}(q)$, $H_{2} \in \Gamma_{N}(q+t)$, для которых выполняется равенство $G=H_{1} \cdot H_{2}$.

Доказательство. В силу сделанных выше замечаний $G \in \Gamma_{N}(q-1) \cdot \Gamma_{N}(q+t+1)$, то есть существуют подстановки $R_{1} \in \Gamma_{N}(q-1)$, $R_{2} \in \Gamma_{N}(q+t+1)$, для которых выполняется равенство $G=R_{1} \cdot R_{2}$. При этом очевидно, что $\left|\Gamma\left(R_{1}\right) \cap \Gamma\left(R_{2}\right)\right| \geq 2$.

Основная идея доказательства заключается в том, чтобы построить такую подстановку $T$, что $R_{1} T \in \Gamma_{N}(q), T^{-1} R_{2} \in \Gamma_{N}(q+t)$. Тогда для подстановок $H_{1}=R_{1} T, H_{2}=T^{-1} R_{2}$ выполняется искомое равенство $G=H_{1} \cdot H_{2}$.

Обозначим

$$
\begin{gathered}
R_{1}=\left(\alpha_{1}^{(1)}, \ldots, \alpha_{m_{1}}^{(1)}\right)\left(\beta_{1}^{(1)}, \ldots, \beta_{m_{2}}^{(1)}\right) \ldots\left(\gamma_{1}^{(1)}, \ldots, \gamma_{m_{s}}^{(1)}\right), \quad \sum_{i=1}^{s} m_{i}=q-1, \\
R_{2}=\left(\alpha_{1}^{(2)}, \ldots, \alpha_{t_{1}}^{(2)}\right)\left(\beta_{1}^{(2)}, \ldots, \beta_{t_{2}}^{(2)}\right) \ldots\left(\gamma_{1}^{(2)}, \ldots, \gamma_{t_{r}}^{(2)}\right), \quad \sum_{i=1}^{r} t_{i}=q+t+1 .
\end{gathered}
$$

Случай 1. Пусть в подстановке $R_{2}$ существует неединичный цикл длины больше 2 , не все точки которого входят в $\Gamma\left(R_{1}\right)$. Точнее, пусть $t_{1} \geq 3$, $\alpha_{1}^{(2)}=\alpha_{1}^{(1)}$ и $\alpha_{t_{1}}^{(2)} \notin \Gamma\left(R_{1}\right)$. Тогда положим $T=\left(\alpha_{t_{1}}^{(2)}, \alpha_{1}^{(1)}\right)$ и получим

$$
\begin{aligned}
R_{1} T & =\left(\alpha_{1}^{(1)}, \ldots, \alpha_{m_{1}}^{(1)}\right)\left(\beta_{1}^{(1)}, \ldots, \beta_{m_{2}}^{(1)}\right) \ldots\left(\gamma_{1}^{(1)}, \ldots, \gamma_{m_{s}}^{(1)}\right) \cdot\left(\alpha_{t_{1}}^{(2)}, \alpha_{1}^{(1)}\right)= \\
= & \left(\alpha_{1}^{(1)}, \ldots, \alpha_{m_{1}}^{(1)}, \alpha_{t_{1}}^{(2)}\right)\left(\beta_{1}^{(1)}, \ldots, \beta_{m_{2}}^{(1)}\right) \ldots\left(\gamma_{1}^{(1)}, \ldots, \gamma_{m_{s}}^{(1)}\right) \in \Gamma_{N}(q), \\
T^{-1} R_{2} & =\left(\alpha_{t_{1}}^{(2)}, \alpha_{1}^{(1)}\right) \cdot\left(\alpha_{1}^{(2)}, \ldots, \alpha_{t_{1}}^{(2)}\right)\left(\beta_{1}^{(2)}, \ldots, \beta_{t_{2}}^{(2)}\right) \ldots\left(\gamma_{1}^{(2)}, \ldots, \gamma_{t_{r}}^{(2)}\right)= \\
& =\left(\alpha_{2}^{(2)}, \ldots, \alpha_{t_{1}}^{(2)}\right)\left(\beta_{1}^{(2)}, \ldots, \beta_{t_{2}}^{(2)}\right) \ldots\left(\gamma_{1}^{(2)}, \ldots, \gamma_{t_{r}}^{(2)}\right) \in \Gamma_{N}(q+t) .
\end{aligned}
$$


Случай 2. Пусть в подстановке $R_{2}$ существуют неединичные циклы, не все точки которых входят в $\Gamma\left(R_{1}\right)$, и все такие циклы являются транспозициями. Точнее, пусть $t_{1}=2, \alpha_{1}^{(2)}=\alpha_{1}^{(1)}$ и $\alpha_{2}^{(2)} \notin \Gamma\left(R_{1}\right)$. По условию существует еще одна точка (кроме $\left.\alpha_{1}^{(2)}\right)$, принадлежащая $\Gamma\left(R_{1}\right) \cap \Gamma\left(R_{2}\right)$. Пусть $\beta_{1}^{(2)} \in \Gamma\left(R_{1}\right) \cap \Gamma\left(R_{2}\right)$.

2.1. Пусть $\quad R_{1}\left(\alpha_{1}^{(2)}\right) \neq \beta_{1}^{(2)}, \quad R_{1}\left(\beta_{1}^{(2)}\right) \neq \alpha_{1}^{(2)} . \quad$ Тогда $\quad$ положим $T=\left(\alpha_{1}^{(2)}, \beta_{1}^{(2)}\right)$ и получим

$$
\begin{gathered}
R_{1}^{\prime}=R_{1} T \in \Gamma_{N}(q-1), \alpha_{2}^{(2)} \notin \Gamma\left(R_{1}^{\prime}\right), \\
R_{2}^{\prime}=T^{-1} R_{2}=\left(\alpha_{1}^{(2)}, \beta_{1}^{(2)}\right) \cdot\left(\alpha_{1}^{(2)}, \alpha_{2}^{(2)}\right)\left(\beta_{1}^{(2)}, \ldots, \beta_{t_{2}}^{(2)}\right) \ldots\left(\gamma_{1}^{(2)}, \ldots, \gamma_{t_{r}}^{(2)}\right)= \\
=\left(\alpha_{1}^{(2)}, \beta_{2}^{(2)}, \ldots, \beta_{t_{2}}^{(2)}, \beta_{1}^{(2)}, \alpha_{2}^{(2)}\right) \ldots\left(\gamma_{1}^{(2)}, \ldots, \gamma_{t_{r}}^{(2)}\right) \in \Gamma_{N}(q+t+1), \\
G=R_{1}^{\prime} \cdot R_{2}^{\prime} .
\end{gathered}
$$

Видно, что подстановки $R_{1}^{\prime}, R_{2}{ }_{2}$ удовлетворяют условиям случая 1 .

2.2. Пусть теперь $m_{1} \geq 3, \quad R_{1}\left(\alpha_{1}^{(2)}\right)=\beta_{1}^{(2)} \quad$ (то есть $\left.\quad \alpha_{2}^{(1)}=\beta_{1}^{(2)}\right)$ или $R_{1}\left(\beta_{1}^{(2)}\right)=\alpha_{1}^{(2)} \quad$ (то есть $\left.\quad \alpha_{m_{1}}^{(1)}=\beta_{1}^{(2)}\right) . \quad$ В $\quad$ первом $\quad$ случае $\quad$ положим $T=\left(\alpha_{1}^{(2)}, \beta_{1}^{(2)}, \alpha_{2}^{(2)}\right)$ и получим

$$
\begin{gathered}
R_{1} T=\left(\alpha_{1}^{(1)}, \beta_{1}^{(2)}, \ldots, \alpha_{m_{1}}^{(1)}\right)\left(\beta_{1}^{(1)}, \ldots, \beta_{m_{2}}^{(1)}\right) \ldots\left(\gamma_{1}^{(1)}, \ldots, \gamma_{m_{s}}^{(1)}\right) \cdot\left(\alpha_{1}^{(1)}, \beta_{1}^{(2)}, \alpha_{2}^{(2)}\right)= \\
=\left(\alpha_{1}^{(1)}, \alpha_{2}^{(2)}\right)\left(\beta_{1}^{(2)}, \ldots, \alpha_{m_{1}}^{(1)}\right)\left(\beta_{1}^{(1)}, \ldots, \beta_{m_{2}}^{(1)}\right) \ldots\left(\gamma_{1}^{(1)}, \ldots, \gamma_{m_{s}}^{(1)}\right) \in \Gamma_{N}(q), \\
T^{-1} R_{2}=\left(\alpha_{1}^{(2)}, \alpha_{2}^{(2)}, \beta_{1}^{(2)}\right) \cdot\left(\alpha_{1}^{(2)}, \alpha_{2}^{(2)}\right)\left(\beta_{1}^{(2)}, \ldots, \beta_{t_{2}}^{(2)}\right) \ldots\left(\gamma_{1}^{(2)}, \ldots, \gamma_{t_{r}}^{(2)}\right)= \\
=\left(\alpha_{2}^{(2)}, \beta_{2}^{(2)}, \ldots, \beta_{t_{2}}^{(2)}, \beta_{1}^{(2)}\right) \ldots\left(\gamma_{1}^{(2)}, \ldots, \gamma_{t_{r}}^{(2)}\right) \in \Gamma_{N}(q+t) .
\end{gathered}
$$

Во втором случае положим $T=\left(\alpha_{1}^{(2)}, \alpha_{2}^{(2)}, \beta_{1}^{(2)}\right)$ и получим аналогичный результат:

$$
\begin{aligned}
R_{1} T & =\left(\alpha_{1}^{(1)}, \ldots, \alpha_{m_{1}-1}^{(1)}, \beta_{1}^{(2)}\right)\left(\beta_{1}^{(1)}, \ldots, \beta_{m_{2}}^{(1)}\right) \ldots\left(\gamma_{1}^{(1)}, \ldots, \gamma_{m_{s}}^{(1)}\right) \cdot\left(\alpha_{1}^{(1)}, \alpha_{2}^{(2)}, \beta_{1}^{(2)}\right)= \\
& =\left(\alpha_{1}^{(1)}, \ldots, \alpha_{m_{1}-1}^{(1)}\right)\left(\beta_{1}^{(2)}, \alpha_{2}^{(2)}\right)\left(\beta_{1}^{(1)}, \ldots, \beta_{m_{2}}^{(1)}\right) \ldots\left(\gamma_{1}^{(1)}, \ldots, \gamma_{m_{s}}^{(1)}\right) \in \Gamma_{N}(q),
\end{aligned}
$$




$$
\begin{aligned}
T^{-1} R_{2} & =\left(\alpha_{1}^{(2)}, \beta_{1}^{(2)}, \alpha_{2}^{(2)}\right) \cdot\left(\alpha_{1}^{(2)}, \alpha_{2}^{(2)}\right)\left(\beta_{1}^{(2)}, \ldots, \beta_{t_{2}}^{(2)}\right) \ldots\left(\gamma_{1}^{(2)}, \ldots, \gamma_{t_{r}}^{(2)}\right)= \\
& =\left(\alpha_{1}^{(2)}, \beta_{2}^{(2)}, \ldots, \beta_{t_{2}}^{(2)}, \beta_{1}^{(2)}\right) \ldots\left(\gamma_{1}^{(2)}, \ldots, \gamma_{t_{r}}^{(2)}\right) \in \Gamma_{N}(q+t) .
\end{aligned}
$$

2.3. Пусть $\quad m_{1}=2, \quad R_{1}\left(\alpha_{1}^{(2)}\right)=\beta_{1}^{(2)} \quad$ или $\quad R_{1}\left(\beta_{1}^{(2)}\right)=\alpha_{1}^{(2)} \quad$ (то есть $\left.R_{1}=\left(\alpha_{1}^{(1)}, \beta_{1}^{(2)}\right)\left(\beta_{1}^{(1)}, \ldots, \beta_{m_{2}}^{(1)}\right) \ldots\left(\gamma_{1}^{(1)}, \ldots, \gamma_{m_{s}}^{(1)}\right)\right) . \quad$ Также предположим, $\quad$ что $\beta_{2}^{(2)} \in \Gamma\left(R_{1}\right)$. Положим $T=\left(\alpha_{2}^{(2)}, \beta_{1}^{(2)}, \beta_{2}^{(2)}\right)$ и получим

$$
\begin{gathered}
R_{1} T=\left(\alpha_{1}^{(1)}, \beta_{1}^{(2)}\right)\left(\beta_{1}^{(1)}, \ldots, \beta_{m_{2}}^{(1)}\right) \ldots\left(\gamma_{1}^{(1)}, \ldots, \gamma_{m_{s}}^{(1)}\right) \cdot\left(\alpha_{2}^{(2)}, \beta_{1}^{(2)}, \beta_{2}^{(2)}\right) \in \Gamma_{N}(q), \\
T^{-1} R_{2}=\left(\alpha_{2}^{(2)}, \beta_{2}^{(2)}, \beta_{1}^{(2)}\right) \cdot\left(\alpha_{1}^{(2)}, \alpha_{2}^{(2)}\right)\left(\beta_{1}^{(2)}, \beta_{2}^{(2)}, \ldots, \beta_{t_{2}}^{(2)}\right) \ldots\left(\gamma_{1}^{(2)}, \ldots, \gamma_{t_{r}}^{(2)}\right)= \\
=\left(\alpha_{1}^{(2)}, \alpha_{2}^{(2)}, \beta_{3}^{(2)}, \ldots, \beta_{t_{2}}^{(2)}, \beta_{1}^{(2)}\right) \ldots\left(\gamma_{1}^{(2)}, \ldots, \gamma_{t_{r}}^{(2)}\right) \in \Gamma_{N}(q+t) .
\end{gathered}
$$

2.4. Пусть $\quad m_{1}=2, \quad R_{1}\left(\alpha_{1}^{(2)}\right)=\beta_{1}^{(2)} \quad$ или $\quad R_{1}\left(\beta_{1}^{(2)}\right)=\alpha_{1}^{(2)} \quad$ (то $\quad$ есть $\left.R_{1}=\left(\alpha_{1}^{(1)}, \beta_{1}^{(2)}\right)\left(\beta_{1}^{(1)}, \ldots, \beta_{m_{2}}^{(1)}\right) \ldots\left(\gamma_{1}^{(1)}, \ldots, \gamma_{m_{s}}^{(1)}\right)\right)$. Предположим, что $\beta_{2}^{(2)} \notin \Gamma\left(R_{1}\right)$. Тогда по условию случая 2 имеем $t_{2}=2$, то есть

$$
R_{2}=\left(\alpha_{1}^{(2)}, \alpha_{2}^{(2)}\right)\left(\beta_{1}^{(2)}, \beta_{2}^{(2)}\right) \ldots\left(\gamma_{1}^{(2)}, \ldots, \gamma_{t_{r}}^{(2)}\right) .
$$

Кроме того, найдется точка $\beta_{1}^{(1)} \in \Gamma\left(R_{1}\right), \quad \beta_{1}^{(1)} \notin \Gamma\left(R_{2}\right)$. Действительно, в противном случае $\Gamma\left(R_{1}\right) \subset \Gamma\left(R_{2}\right)$, и при $q>4$ получаем противоречие:

$$
|\Gamma(G)|=\left|\Gamma\left(R_{1} R_{2}\right)\right| \leq\left|\Gamma\left(R_{2}\right)\right|=q+t+1<2 q+t-3 .
$$

В этом случае положим $T=\left(\alpha_{1}^{(2)}, \alpha_{2}^{(2)}\right)\left(\beta_{1}^{(2)}, \beta_{1}^{(1)}\right)$ и получим

$$
\begin{gathered}
R_{1} T=\left(\alpha_{1}^{(1)}, \beta_{1}^{(2)}\right)\left(\beta_{1}^{(1)}, \ldots, \beta_{m_{2}}^{(1)}\right) \ldots\left(\gamma_{1}^{(1)}, \ldots, \gamma_{m_{s}}^{(1)}\right) \cdot\left(\alpha_{1}^{(1)}, \alpha_{2}^{(2)}\right)\left(\beta_{1}^{(2)}, \beta_{1}^{(1)}\right)= \\
=\left(\alpha_{1}^{(1)}, \beta_{1}^{(1)}, \ldots, \beta_{m_{2}}^{(1)}, \beta_{1}^{(2)}, \alpha_{2}^{(2)}\right) \ldots\left(\gamma_{1}^{(1)}, \ldots, \gamma_{m_{s}}^{(1)}\right) \in \Gamma_{N}(q), \\
T^{-1} R_{2}=\left(\alpha_{1}^{(2)}, \alpha_{2}^{(2)}\right)\left(\beta_{1}^{(2)}, \beta_{1}^{(1)}\right) \cdot\left(\alpha_{1}^{(2)}, \alpha_{2}^{(2)}\right)\left(\beta_{1}^{(2)}, \beta_{2}^{(2)}\right) \ldots\left(\gamma_{1}^{(2)}, \ldots, \gamma_{t_{r}}^{(2)}\right)= \\
=\left(\beta_{1}^{(2)}, \beta_{1}^{(1)}, \beta_{2}^{(2)}\right) \ldots\left(\gamma_{1}^{(2)}, \ldots, \gamma_{t_{r}}^{(2)}\right) \in \Gamma_{N}(q+t) .
\end{gathered}
$$

Осталось рассмотреть последний возможный случай.

Случай 3. Для любого неединичного цикла подстановки $R_{2}$ либо все точки этого цикла принадлежат $\Gamma\left(R_{1}\right)$, либо все точки этого цикла 
не принадлежат $\Gamma\left(R_{1}\right)$. Поскольку $\left|\Gamma\left(R_{1}\right) \cap \Gamma\left(R_{2}\right)\right| \geq 2, \quad$ то существует цикл $\left(\alpha_{1}^{(2)}, \ldots, \alpha_{t_{1}}^{(2)}\right), \quad$ удовлетворяющий первому условию (то есть $\left.\alpha_{1}^{(2)}, \ldots, \alpha_{t_{1}}^{(2)} \in \Gamma\left(R_{1}\right)\right)$. В то же время существует цикл, удовлетворяющий второму условию. Действительно, если это не так, то получаем противоречие $\Gamma\left(R_{2}\right) \subset \Gamma\left(R_{1}\right)$ и

$$
\left|\Gamma\left(R_{2}\right)\right|=q+t+1 \quad>\quad q-1=\left|\Gamma\left(R_{1}\right)\right| .
$$

Будем считать, что $\beta_{1}^{(2)}, \ldots, \beta_{t_{2}}^{(2)} \notin \Gamma\left(R_{1}\right)$.

3.1. Пусть существует $j \in\left\{1, \ldots, t_{1}\right\}$ со свойством $R_{1}\left(\alpha_{j}^{(2)}\right) \neq \alpha_{j-1}^{(2)}$. Тогда положим $T=\left(\alpha_{j}^{(2)}, \beta_{1}^{(2)}, \alpha_{j-1}^{(2)}\right)$ и получим

$$
\begin{gathered}
R_{1} T=R_{1} \cdot\left(\alpha_{j}^{(2)}, \beta_{1}^{(2)}, \alpha_{j-1}^{(2)}\right) \in \Gamma_{N}(q), \\
T^{-1} R_{2}=\left(\alpha_{j}^{(2)}, \alpha_{j-1}^{(2)}, \beta_{1}^{(2)}\right) \cdot\left(\alpha_{1}^{(2)}, \ldots, \alpha_{t_{1}}^{(2)}\right)\left(\beta_{1}^{(2)}, \ldots, \beta_{t_{2}}^{(2)}\right) \ldots\left(\gamma_{1}^{(2)}, \ldots, \gamma_{t_{r}}^{(2)}\right)= \\
=\left(\alpha_{1}^{(2)}, \ldots, \alpha_{j-1}^{(2)}, \beta_{2}^{(2)}, \ldots, \beta_{t_{2}}^{(2)}, \beta_{1}^{(2)}, \alpha_{j+1}^{(2)}, \ldots, \alpha_{t_{1}}^{(2)}\right) \ldots\left(\gamma_{1}^{(2)}, \ldots, \gamma_{t_{r}}^{(2)}\right) \in \Gamma_{N}(q+t) .
\end{gathered}
$$

3.2. Пусть $R_{1}\left(\alpha_{j}^{(2)}\right)=\alpha_{j-1}^{(2)}$ для всех $j \in\left\{1, \ldots, t_{1}\right\}$. Это означает, что в подстановке $R_{1}$ имеется неединичный цикл $\left(\alpha_{1}^{(2)}, \alpha_{t_{1}}^{(2)}, \alpha_{t_{1}-1}^{(2)}, \ldots, \alpha_{2}^{(2)}\right)=$ $=\left(\alpha_{1}^{(2)}, \ldots, \alpha_{t_{1}}^{(2)}\right)^{-1}$. Кроме того, выше отмечалось, что включение $\Gamma\left(R_{1}\right) \subset \Gamma\left(R_{2}\right)$ противоречит условию леммы. Значит, в подстановке $R_{1}$ имеется неединичный цикл $\left(\gamma_{1}^{(1)}, \ldots, \gamma_{m_{s}}^{(1)}\right)$ со свойством $\gamma_{1}^{(1)} \notin \Gamma\left(R_{2}\right)$.

Если $t_{1} \geq 3$, то положим $T=\left(\alpha_{1}^{(2)}, \alpha_{2}^{(2)}\right)\left(\beta_{1}^{(2)}, \gamma_{1}^{(1)}\right)$ и получим

$$
\begin{gathered}
R_{1}^{\prime}=R_{1} T=\left(\alpha_{1}^{(2)}, \alpha_{t_{1}}^{(2)}, \ldots, \alpha_{2}^{(2)}\right)\left(\beta_{1}^{(1)}, \ldots, \beta_{m_{2}}^{(1)}\right) \ldots\left(\gamma_{1}^{(1)}, \ldots, \gamma_{m_{s}}^{(1)}\right) \cdot\left(\alpha_{1}^{(2)}, \alpha_{2}^{(2)}\right)\left(\beta_{1}^{(2)}, \gamma_{1}^{(1)}\right)= \\
=\left(\alpha_{1}^{(2)}, \alpha_{t_{1}}^{(2)}, \alpha_{t_{1}-1}^{(2)}, \ldots, \alpha_{3}^{(2)}\right)\left(\beta_{1}^{(1)}, \ldots, \beta_{m_{2}}^{(1)}\right) \ldots\left(\gamma_{1}^{(1)}, \ldots, \gamma_{m_{s}}^{(1)}, \beta_{1}^{(2)}\right) \in \Gamma_{N}(q-1), \\
R_{2}^{\prime}=T^{-1} R_{2}=\left(\alpha_{1}^{(2)}, \alpha_{2}^{(2)}\right)\left(\beta_{1}^{(2)}, \gamma_{1}^{(1)}\right) \cdot\left(\alpha_{1}^{(2)}, \ldots, \alpha_{t_{1}}^{(2)}\right)\left(\beta_{1}^{(2)}, \ldots, \beta_{t_{2}}^{(2)}\right) \ldots\left(\gamma_{1}^{(2)}, \ldots, \gamma_{t_{r}}^{(2)}\right)= \\
=\left(\alpha_{1}^{(2)}, \alpha_{3}^{(2)}, \ldots, \alpha_{t_{1}}^{(2)}\right)\left(\beta_{1}^{(2)}, \gamma_{1}^{(1)}, \beta_{2}^{(2)}, \ldots, \beta_{t_{2}}^{(2)}\right) \ldots\left(\gamma_{1}^{(2)}, \ldots, \gamma_{t_{r}}^{(2)}\right) \in \Gamma_{N}(q+t+1), \\
G=R_{1}^{\prime} \cdot R_{2}^{\prime} .
\end{gathered}
$$

При этом подстановки $R_{1}^{\prime}, R_{2}^{\prime}$ удовлетворяют условиям случая 1. 
Если же $t_{1}=2$, то выберем точку $\delta \notin \Gamma\left(R_{1}\right) \cup \Gamma\left(R_{2}\right)$. Такая точка существует ввиду условия $2 q+t-2<N$ леммы. Положим $T=\left(\alpha_{1}^{(2)}, \alpha_{2}^{(2)}\right) \times$ $\times\left(\beta_{1}^{(2)}, \gamma_{1}^{(1)}, \delta\right)$ и получим

$$
\begin{gathered}
R_{1}^{\prime}=R_{1} T=\left(\alpha_{1}^{(2)}, \alpha_{2}^{(2)}\right)\left(\beta_{1}^{(1)}, \ldots, \beta_{m_{2}}^{(1)}\right) \ldots\left(\gamma_{1}^{(1)}, \ldots, \gamma_{m_{s}}^{(1)}\right) \cdot\left(\alpha_{1}^{(2)}, \alpha_{2}^{(2)}\right)\left(\beta_{1}^{(2)}, \gamma_{1}^{(1)}, \delta\right)= \\
=\left(\beta_{1}^{(1)}, \ldots, \beta_{m_{2}}^{(1)}\right) \ldots\left(\gamma_{1}^{(1)}, \ldots, \gamma_{m_{s}}^{(1)}, \delta, \beta_{1}^{(2)}\right) \in \Gamma_{N}(q-1), \\
R_{2}^{\prime}=T^{-1} R_{2}=\left(\alpha_{1}^{(2)}, \alpha_{2}^{(2)}\right)\left(\beta_{1}^{(2)}, \delta, \gamma_{1}^{(1)}\right) \cdot\left(\alpha_{1}^{(2)}, \alpha_{2}^{(2)}\right)\left(\beta_{1}^{(2)}, \ldots, \beta_{t_{2}}^{(2)}\right) \ldots\left(\gamma_{1}^{(2)}, \ldots, \gamma_{t_{r}}^{(2)}\right)= \\
=\left(\beta_{1}^{(2)}, \delta, \gamma_{1}^{(1)}, \beta_{2}^{(2)}, \ldots, \beta_{t_{2}}^{(2)}\right) \ldots\left(\gamma_{1}^{(2)}, \ldots, \gamma_{t_{r}}^{(2)}\right) \in \Gamma_{N}(q+t+1), \\
G=R_{1}^{\prime} \cdot R_{2}^{\prime} .
\end{gathered}
$$

При этом снова подстановки $R_{1}^{\prime}, R_{2}^{\prime}$ удовлетворяют условиям случая 1 . Лемма 4 и теорема 2 доказаны.

Следствие. При $N>10,2 \leq t<N-2,2 \leq q<\frac{N-t}{2}+1$

$$
\Gamma_{N}(q) \cdot \Gamma_{N}(q+t) \supseteq \bigcup_{r=t}^{2 q+t-2} \Gamma_{N}(r) .
$$

Далее рассмотрим, какие подстановки из множеств $\Gamma_{N}(2 q+t-1)$, $\Gamma_{N}(2 q+t)$ принадлежат произведению $\Gamma_{N}(q) \cdot \Gamma_{N}(q+t)$. Пусть $G$ является произведением $r$ неединичных циклов, длины которых равны $m_{1}, m_{2}, \ldots, m_{r}$.

Утверждение 5. Пусть $N>10,2 \leq t<N-2,2 \leq q<\frac{N-t}{2}+1$, подстановка $G \in \Gamma_{N}(2 q+t)$ является произведением $r$ неединичных изиклов, длины которых равны $m_{1}, m_{2}, \ldots, m_{r}, \sum_{i=1}^{r} m_{i}=2 q+t$. Подстановка $G$ принадлежсит множеству $\Gamma_{N}(q) \cdot \Gamma_{N}(q+t)$ в том и только в том случае, когда существует такое подмножество $\left\{i_{1}, \ldots, i_{k}\right\} \subseteq\{1, \ldots, r\}$, что $m_{i_{1}}+\ldots+m_{i_{k}}=q$.

Доказательство полностью аналогично доказательству утверждения 3.

Замечание. Из доказанного утверждения вытекает, что $\Gamma_{N}(2 q+t) \not \subset \Gamma_{N}(q) \cdot \Gamma_{N}(q+t)$. Действительно, $\quad(2 q+t)$-циклы лежат в $\Gamma_{N}(2 q+t)$, однако они не удовлетворяют условию утверждения 11 . 
Утверждение 6. Пусть $N>10,2 \leq t<N-2,2 \leq q<\frac{N-t}{2}+1$, подстановка $G \in \Gamma_{N}(2 q+t-1)$ является произведением $r$ неединичныхх ииклов, длины которых равны $m_{1}, m_{2}, \ldots, m_{r}, \sum_{i=1}^{r} m_{i}=2 q+t-1$. Подстановка $G$ принадлежит множеству $\Gamma_{N}(q) \cdot \Gamma_{N}(q+t)$ в том и только в том случае, когда существуют такое $i_{0} \in\{1, \ldots, r\} \quad$ и такое подмножество $\left\{i_{1}, \ldots, i_{k}\right\} \subseteq\{1, \ldots, r\} \backslash\left\{i_{0}\right\}$, что $m_{i_{0}}>2$ и $q-m_{i_{1}}+m_{i_{2}} \ldots+m_{i_{k}} \in\left\{2, \ldots, m_{i_{0}}-1\right\}$.

Доказательство полностью аналогично доказательству утверждения 4.

Замечание. Из доказанного утверждения вытекает, что в общем случае $\Gamma_{N}(2 q+t-1) \not \subset \Gamma_{N}(q) \cdot \Gamma_{N}(q+t)$.

Действительно, пусть $2 q+t-1=2 d-$ четное число. Тогда в $\Gamma_{N}(2 q+t-1)$ содержатся подстановки, являющиеся произведением $d$ транспозиций. В обозначениях утверждения 6 имеем $r=d, m_{1}=m_{2}=\ldots=m_{d}=2$. Следовательно, не существует $i_{0} \in\{1, \ldots, d\}$ со свойством $m_{i_{0}}>2$, и такие подстановки не удовлетворяют условию утверждения 6.

Итак, в теореме 2, утверждениях 5, 6 полностью описано строение множества $\Gamma_{N}(q) \cdot \Gamma_{N}(q+t)$ при $2 \leq t<N-2,2 \leq q<\frac{N-t}{2}+1$.

\section{Список литературы}

1. Пичкур А. Б. Описание класса подстановок, представимых в виде произведения двух подстановок с фиксированным числом мобильных точек // Математические вопросы криптографии. — 2012. - Т. 3. Вып. 2. C. $79-95$. 
